\title{
RECURSIVE PROPERTIES OF TRANSFORMATION GROUPS. II
}

\author{
w. H. GOTTSCHALK
}

The purpose of this note is to sharpen a previous result on the transmission of recursive properties of a transformation group to certain of its subgroups. [See Recursive properties of transformation groups, by W. H. Gottschalk and G. A. Hedlund, Bull. Amer. Math. Soc. vol. 52 (1946) pp. 637-641.]

Let $T$ be a multiplicative topological group with identity $e$. A subset $R$ of $T$ is said to be relatively dense provided that $T=R K$ for some compact set $K$ in $T$.

Lemma 1. If $R$ is a relatively dense closed semi-group $(R R \subset R)$ in $T$, then $R$ is a subgroup of $T$.

Proof. Suppose $r \in R$ and $U$ is a neighborhood of $e$. It is sufficient to show that $r^{-1} U \cap R \neq \varnothing$. Let $V$ be a neighborhood of $e$ for which $V V^{-1} \subset U$ and let $K$ be a compact set in $T$ for which $T=R K$. There exists a finite collection $F$ of right translates of $V$ which covers $K$. Choose $k_{0} \in K$. Now $r^{-1} k_{0}=r_{1} k_{1}$ for some $r_{1} \in R$ and some $k_{1} \in K$. Again $r^{-1} k_{1}=r_{2} k_{2}$ for some $r_{2} \in R$ and some $k_{2} \in K$. This may be continued. Thus there exist sequences $k_{0}, k_{1}, \cdots$ in $K$ and $r_{1}, r_{2}, \cdots$ in $R$ such that $r^{-1} k_{i}=r_{i+1} k_{i+1}(i=0,1, \cdots)$. Select integers $m$ and $n(0 \leqq m<n)$ and an element $V_{0}$ of $F$ such that $k_{m}, k_{n} \in V_{0}$. Now $r^{-1} k_{m} k_{n}{ }^{-1}$ $=\left(r^{-1} k_{m} k_{m+1}^{-1}\right)\left(k_{m+1} k_{m+2}^{-1}\right) \cdots\left(k_{n-1} k_{n}^{-1}\right)=r_{m+1} r_{m+2} \cdots r r_{n} \in R$. Also $r^{-1} k_{m} k_{n}^{-1} \in r^{-1} V_{0} V_{0}^{-1} \subset r^{-1} V V^{-1} \subset r^{-1} U$. Hence $r^{-1} U \cap R \neq \varnothing$ and the proof is completed.

Now let $T$ act as a transformation group on a topological space $X$. That is to say, suppose that to $x \in X$ and $t \in T$ is assigned a point, denoted $x t$, of $X$ such that: (1) $x e=x(x \in X) ;(2)(x t) s=x(t s)(x \in X ; t$, $s \in T)$; (3) The function $x t$ defines a continuous transformation of $X \times T$ into $X$. We assume for the remainder of the paper that $x$ is a fixed point of $X, T$ is locally compact and $S$ is a relatively dense invariant subgroup of $T$. Let $\Sigma$ denote the maximal subset of $T$ for which $x \Sigma \subset(x S)^{*}$ where the star denotes the closure operator.

LEMma 2. The set $\Sigma$ is a closed subgroup of $T$ which contains $S$.

Proof. Obviously $\Sigma \supset S$. From $x \Sigma^{*} \subset(x \Sigma)^{*} \subset(x S)^{*}$ we conclude that $\Sigma$ is closed. By Lemma 1 it is now enough to show that $\Sigma$ is a

Presented to the Society, September 2, 1947; received by the editors June 30, 1947. 
semi-group. Suppose $\sigma, \tau \in \Sigma$. From $x \sigma \in(x S)^{*}$ it follows that $x \sigma \tau$ $\in(x S)^{*} \tau \subset(x S \tau)^{*} \subset(x \tau S)^{*}$. From $x \tau \in(x S)^{*}$ it follows that $x \tau S$ $\subset(x S)^{*} S \subset(x S S)^{*} \subset(x S)^{*}$. Hence $x \sigma \tau \in(x S)^{*}$. Thus $\sigma \tau \in \Sigma$ and the proof is completed.

Lemma 3. If $W$ is a neighborhood of e, then $x \notin(x[T-\Sigma W]) *$.

Proof. We first show that if $t \in T-\Sigma$, then $x \notin\left(x \Sigma V_{0}\right)^{*}$ for some neighborhood $V_{0}$ of $t$. Suppose $t \in T-\Sigma$. Since $t^{-1} \notin \Sigma$ by Lemma 2, $x t^{-1} \oplus(x \Sigma)^{*}$ and $x \notin(x \Sigma t)^{*}$. There are neighborhoods $U$ of $x$ and $V$ of $e$ such that $V=V^{-1}$ and $U V \cap x \Sigma t=\varnothing$. It follows that $U \cap x \Sigma t V$ $=\varnothing$. Define $V_{0}=t V$.

We may assume $W$ is open. Define $N=K-\Sigma W$ where $K$ is a compact set in $T$ such that $T=S K$. Using Lemma 2 we conclude that $T=S K \subset S(N \cup \Sigma W) \subset S N \cup S \Sigma W \subset \Sigma N \cup \Sigma W$ and $\Sigma N \cap \Sigma W$ $=\varnothing$. Hence $T-\Sigma W=\Sigma N$. By the preceding paragraph, to each $n \in N$ there corresponds a neighborhood $V_{n}$ of $n$ such that $x \notin\left(x \Sigma V_{n}\right)^{*}$. Since finitely many of the $V_{n}$ cover $N, x \in(x \Sigma N)^{*}$. The proof is completed.

Lemma 4. If $U$ is a neighborhood of $x$, then there exists a compact set $M$ in $T$ such that $x M \subset U$ and $\Sigma \subset S M^{-1}$.

Proof. Define $N=K \cap \Sigma$ where $K$ is a compact set in $T$ such that $T=S K$. If $n \in N$, then $x n \in(x S)^{*}$ and $x \in\left(x S n^{-1}\right)^{*}$. Thus $n \in N$ implies the existence of $s_{n} \in S$ such that $x s_{n} n^{-1} \in i n t U$ and hence the existence of a compact neighborhood $W_{n}$ of $s_{n} n^{-1}$ such that $x W_{n} \subset U$. Since $N$ is compact by Lemma 2 , there is a finite subset $F$ of $N$ for which $N \subset \mathrm{U}_{n \in F} W_{n}^{-1} s_{n}$. Define $M=\bigcup_{n \in F} W_{n}$. Clearly $x M \subset U$. Using Lemma 2 we conclude that $\Sigma \subset S N \subset S M^{-1}$. The proof is completed.

Let there be distinguished in $T$ certain sets, called admissible, which satisfy this condition: If $A$ is an admissible set and if $B$ is a set in $T$ such that $A \subset B K$ for some compact set $K$ in $T$, then $B$ is an admissible set. A subgroup $R$ of $T$ is said to be recursive at $x$ provided that to each neighborhood $U$ of $x$ there corresponds an admissible set $A$ such that $A \subset R$ and $x A \subset U$.

LEMMA 5. If $T$ is recursive at $x$, then $\Sigma$ is recursive at $x$.

Proof. Let $U$ be a neighborhood of $x$. There are neighborhoods $V$ of $x$ and $W$ of $e$ such that $W=W^{-1}, W$ is compact and $V W \subset U$. By Lemma 3 we may suppose that $V \cap x(T-\Sigma W)=\varnothing$. There exists an admissible set $A$ in $T$ such that $x A \subset V$. Clearly $A \subset \Sigma W$ and $x A W \subset U$. Define $B=\Sigma \cap A W$. Since $A \subset B W, B$ is an admissible set. Also $B C \Sigma$ and $x B C U$. The proof is completed. 
LEMMA 6. If $\Sigma$ is recursive at $x$, then $S$ is recursive at $x$.

Proof. Let $U$ be an open neighborhood of $x$. By Lemma 4 there exists a compact set $M$ in $T$ such that $x M \subset U$ and $\Sigma \subset S M^{-1}$. Let $V$ be a neighborhood of $x$ for which $V M \subset U$. There exists an admissible set $A$ such that $A \subset \Sigma$ and $x A \subset V$. Hence $x A M \subset U$. Define $B=S \cap A M$. Since $A \subset B M^{-1}, B$ is an admissible set. Also $B \subset S$ and $x B \subset U$. The proof is completed.

The following theorem is an immediate consequence of Lemmas 5 and 6.

THEOREM. If $T$ is recursive at $x$, then $S$ is recursive at $x$.

An interpretation of admissibility arises if we define an admissible subset of $T$ to be a relatively dense subset of $T$. The term "recursive" is then replaced by "almost periodic." For other applications, see the paper cited above.

University of PenNSYLVAaria

\section{FIXED POINT THEOREMS FOR INTERIOR TRANSFORMATIONS}

\section{O. H. HAMILTON}

If $M$ is a bounded continuum in a Euclidean plane $E$ which does not separate $E$ and $T$ is an interior continuous transformation of $M$ onto a subset of $E$ which contains $M$, does $T$ leave a point of $M$ invariant? It is the purpose of this paper to answer this question in the affirmative for certain types of locally connected continua.

Using a notation introduced by Eilenberg $[2, \text { p. 168 }]^{1}$ a continuum $M$ will be said to have property (b) provided every continuous transformation of $M$ into the unit circle $S$ in the Cartesian plane, with center at $o$, is homotopic to a constant mapping, that is, a transformation which transforms each point of $M$ into a single point of $S$. If $T$ is a continuous transformation of a subset $A$ of the plane $E$ into a subset $B$ of $E$, then for each point $x$ of $A$ let $T^{\prime}(x)$ be the point $y$ of $S$ such that the directed line segment $o y$ is parallel in direction and sense to the directed line segment $x, T(x)$. Then $T^{\prime}$ will be referred to as the transformation of $A$ into $S$ derived from $T$. Such a transformation

Presented to the Society, September 3, 1947; received by the editors June 10, 1947.

${ }_{1}^{1}$ Numbers in brackets refer to the references cited at the end of the paper. 